\title{
PD-LI Expressing Recurrent Clear Cell Carcinoma of the Vulva with Durable Partial Response to Pembrolizumab: A Case Report
}

\author{
Manavi Sachdeva,' Natalie \\ YL Ngoi, (D) ${ }^{2}$ Diana Lim, ${ }^{3}$ \\ Michelle LM Poon, ${ }^{2}$ Yee \\ Liang Thian, ${ }^{4}$ Yi Wan \\ Lim, (D) ${ }^{2}$ Siew Eng Lim, ${ }^{2}$ Pearl \\ Tong, ${ }^{5}$ Jeffrey HY Lum, ${ }^{3}$ \\ Joseph $\mathrm{Ng},{ }^{5}$ Arunachalam \\ Ilancheran, ${ }^{5}$ Efren J \\ Domingo, ${ }^{6}$ Jeffrey JH \\ Low, (iD) 5 David SP Tan (iD) 2,7 \\ 'Department of Medicine, National \\ University Health System Singapore, \\ Singapore, I1 9074, Singapore; \\ ${ }^{2}$ Department of Hematology-Oncology, \\ National University Cancer Institute, \\ Singapore, I 19228, Singapore; \\ ${ }^{3}$ Department of Pathology, National \\ University Health System Singapore, \\ Singapore, I1 9074, Singapore; \\ ${ }^{4}$ Department of Diagnostic Imaging, \\ National University Health System \\ Singapore, Singapore, 119074, Singapore; \\ ${ }^{5}$ Division of Gynecology-Oncology, \\ Department of Obstetrics and \\ Gynecology, National University Hospital \\ Singapore, Singapore, 1 19074, Singapore; \\ ${ }^{6}$ Department of Obstetrics and \\ Gynecology, University of the Philippines \\ College of Medicine, Philippine General \\ Hospital, Manila, I000, Metro Manila, \\ Philippines; ${ }^{7}$ Cancer Science Institute, \\ National University of Singapore, \\ Singapore, I17599, Singapore
}

Correspondence: David SP Tan Department of Hematology-Oncology, National University Cancer Institute, IE Kent Ridge Road, Singapore, I19228, Singapore

Tel +65 6779555

Fax +6567342970

Email david_sP_tan@nuhs.edu.sg
Background: The optimal treatment and molecular landscape of recurrent clear cell carcinoma of the vulva (VCCC) are unknown. No reported data exist regarding the efficacy of anti-programmed death 1 (PD-1) immune checkpoint inhibition in VCCC. We report on a patient with chemotherapy-refractory recurrent $\mathrm{VCCC}$, who was found to have high tumor programmed death-ligand 1 (PD-L1) combined positive score (CPS), and subsequently experienced a durable partial response (PR), after treatment with off-label fifth-line pembrolizumab.

Case Presentation: A forty-year-old Filipino woman presented to our center with recurrent VCCC that had progressed on multiple prior lines of cytotoxic chemotherapy. She had a large $25 \mathrm{~cm}$ fungating left groin tumor causing marked lower limb lymphedema, pain and limited mobility. PD-L1 CPS by immunohistochemistry was 45 . She was treated with offlabel pembrolizumab monotherapy and had a dramatic clinical, biochemical and radiological partial response. The progression-free survival of this patient's VCCC after treatment with pembrolizumab, defined as the time from initiation of pembrolizumab until disease progression (by Response Evaluation Criteria in Solid Tumors (version 1.1)), was 8 months. While receiving pembrolizumab, she was diagnosed with concurrent secondary myelodysplastic syndrome with excess blasts (MDS-EB), thought to be related to her prior exposure to multiple lines of cytotoxic chemotherapy. This eventually progressed to acute myeloid leukemia (AML), leading to her demise. Overall survival from time of initiation of pembrolizumab till death was 16 months.

Conclusion: Pembrolizumab was active in this patient with chemotherapy-refractory VCCC which harbored high PD-L1 CPS. Further studies to determine the role of immune checkpoint blockade in the treatment of VCCC are warranted.

Keywords: vulvar cancer, immune check-point blockade, immunotherapy, clear cell carcinoma

\section{Plain Language Summary}

Vulvar clear cell carcinoma is an exceedingly rare cancer for which optimal treatment is unknown. We present a case of a young woman suffering from this rare cancer, which was proven to be resistant to multiple types of chemotherapy treatment. We found that the biomarker, PD-L1, was highly expressed within the tumor and as a result, treated her with the drug pembrolizumab, which targets this axis. Our patient's tumor had a dramatic improvement after pembrolizumab treatment and her symptoms improved markedly. Unfortunately, she developed myelodysplasia, a type of bone marrow cancer, which was thought to be a side effect of the multiple rounds of chemotherapy which she had undergone 
prior to commencing pembrolizumab. She later demised from complications of myelodysplasia. Earlier knowledge of her tumor PD-L1 expression and the potential benefit from pembrolizumab may have spared her from undergoing prior chemotherapy and its feared side effects.

\section{Background}

Primary clear cell carcinoma of the vulva is extremely rare. Only three previous cases have been reported in the literature. $^{1-3}$ This disease has been reported to arise in two distinct settings, either from transformation within a glandular appendage such as the Bartholin's gland or sweat gland, or from extragonadal endometriosis. ${ }^{1,3-6}$ Unlike squamous cell carcinomas of the vulva and vagina, no clear association with human papilloma virus infection has been reported. ${ }^{7}$ Owing to its rarity, the biologic behavior and genomic make up of VCCC are poorly understood. Furthermore, few clinical trials include this patient population. Across known histotypes, no standard therapy exists for patients with recurrent or metastatic vulvar cancer that is not amenable to locoregional treatment, and management is commonly driven by the approach employed in recurrent or metastatic cervical cancer. ${ }^{8}$ As a result, patients with metastatic vulvar cancer have a reportedly poor prognosis and the optimal therapy remains unknown. ${ }^{9}$ The use of novel therapies such as anti-PD-1 immune check-point inhibitors in recurrent VCCC has not previously been described. We present our experience in treating a patient with recurrent chemotherapy-refractory VCCC whose tumor had a high PD-L1 CPS, with pembrolizumab monotherapy, and describe the molecular characterization of this rare tumor.

\section{Case Presentation}

\section{Patient Characteristics and Prior Therapy}

A forty-year-old Filipino female homemaker presented in her home country in April 2017 with a progressively enlarging vulva mass over three months, which she noticed after undergoing depilation waxing in this area. She was a lifetime non-smoker, was nulliparous and had no known history of endometriosis, sexually transmitted disease or prenatal exposure to diethylstilbestrol. She had not undergone previous Papanicolaou smear or pelvic surgery. She had no past medical history nor family history of note. During the course of her disease, she developed significant morbidity with limited mobility, groin pain and lower limb swelling. From the perspective of a young woman, the physical, social and psychosexual implications of her illness were frustrating and distressing.

Physical examination in the Philippines showed a 12 centimeter $(\mathrm{cm})$ cream-colored, firm polypoid mass over the right anterior labia majora, attached from a base measuring $4 \mathrm{~cm}$ in diameter. Matted bilateral inguinal lymph nodes were palpated, measuring $10 \mathrm{~cm}$ by $9 \mathrm{~cm}$, and $4 \mathrm{~cm}$ by $3 \mathrm{~cm}$ in the left and right inguinal fossae, respectively. On further examination, the urethra, left labia majora, vagina, uterus, adnexa and digital rectal examination were noted to be normal. Computed tomography (CT) scans of the thorax, abdomen and pelvis confirmed enlarged lymph nodes in bilateral inguinofemoral regions, but no distant visceral or nodal metastases. The patient underwent wide local excision of the vulvar mass with bilateral inguinal lymph node dissection in the Philippines. However, residual nodal disease in the left femoral station was deemed surgically inoperable due to its proximity to the femoral vessels, precluding safe dissection. Histological examination showed metastatic clear cell adenocarcinoma. Immunohistochemistry (IHC) was positive for cytokeratin, CAM 5.2 and HNF1- $\beta$, and negative for napsin-A, P40, desmin, SMA, S-100 and Melan-A, with 4 of 23 harvested lymph nodes positive for macrometastases, International Federation of Gynecology and Obstetrics (FIGO) Stage IVA. Post-operative magnetic resonance imaging (MRI) scan of the pelvis showed a residual enhancing heterogeneous left inguinofemoral nodal mass measuring $5 \mathrm{~cm}$ on short axis. The patient received adjuvant chemotherapy with intravenous carboplatin and paclitaxel 3-weekly, followed by radical adjuvant pelvic external beam radiotherapy concurrent with weekly cisplatin, which was completed in October 2017. Post-therapy MRI documented regression in the size of this node to a short axis of $3.2 \mathrm{~cm}$ with no new sites of disease. The patient was subsequently placed on 3 monthly surveillance.

Three months later, she re-presented with clinical enlargement of the left inguinofemoral lymph node, and local progression was again confirmed on MRI. This was deemed surgically unresectable. Between January 2018 to September 2018, the patient received second-line carboplatin plus docetaxel for 5 cycles, third-line gemcitabine plus ifosfamide for 4 cycles and fourth-line weekly paclitaxel for 2 cycles. Chemotherapy was discontinued due to grade 3 fatigue and clinical disease progression with worsening pain over the left groin (Table 1). The patient was referred to our center for a second opinion. 
Table I Clinical Presentation and Treatment Summary

\begin{tabular}{|c|c|}
\hline Date & Presentation and Treatment \\
\hline \multicolumn{2}{|c|}{ Treatment received at another institution } \\
\hline Apr 2017 & $\begin{array}{l}\text { Presented with large left vulvar mass and underwent wide local excision of mass with bilateral inguinal lymph node dissection, } \\
\text { however surgically inoperable residual nodal disease in left femoral station was noted at end of surgery. Pathology confirmed } \\
\text { clear cell adenocarcinoma, final stage was FIGO IVA }\end{array}$ \\
\hline Apr - Jul 2017 & Received adjuvant carboplatin + paclitaxel for 6 cycles \\
\hline Sep - Oct 2017 & Received adjuvant pelvic radiotherapy to the inguinal region and vulva concurrent with weekly cisplatin \\
\hline Jan 2018 & Symptomatic relapse with palpable lymph nodes in left inguinal region \\
\hline Jan - Apr 2018 & $\begin{array}{l}\text { Received second-line carboplatin + docetaxel for } 5 \text { cycles, best response was stable disease but suffered disease progression } \\
\text { after cycle } 5\end{array}$ \\
\hline Apr - Aug 2018 & Received third-line ifosfamide + gemcitabine, best response was partial response but suffered disease progression after cycle 4 \\
\hline Aug - Sep 2018 & Received fourth-line weekly paclitaxel with disease progression after 2 cycles \\
\hline \multicolumn{2}{|c|}{ Transfer of care to our institution } \\
\hline Sep 2018 & $\begin{array}{l}\text { Repeat PET/CT scans showed only local disease in the left groin but no distant metastases. Wedge biopsy was performed on } \\
\text { recurrent tumor in the left groin showing clear cell adenocarcinoma. PD-LI CPS was } 45\end{array}$ \\
\hline Oct 2018 & PET-CT showed new tumor thrombi and worsening pelvic adenopathy. Foundation I genetic report sent \\
\hline $\begin{array}{l}\text { Oct } 2018- \\
\text { Sep } 2019\end{array}$ & Received 16 cycles of fifth-line pembrolizumab. Baseline CAI25 298 \\
\hline Dec 2018 & $\begin{array}{l}\text { Rapid reduction in CAI } 25 \text { to } 9.5 \text { after just } 2 \text { cycles of pembrolizumab. Significant reduction in size of left groin tumor and } \\
\text { improvement in left leg swelling }\end{array}$ \\
\hline Jan 2019 & Developed new neutropenia, grade 3 \\
\hline Feb 2019 & CAI 25 nadir of 4.6 \\
\hline Mar 2019 & Hematology consult and bone marrow examination which diagnosed MDS-EB \\
\hline May 2019 & Nil analgesia requirement and able to walk independently \\
\hline Jun 2019 & Worsening left groin pain; interim PET CT showed left inguinal ulcerative lesion more metabolically active \\
\hline Sep 2019 & Returned to the Philippines, defaulted follow-up in Singapore thereafter \\
\hline Dec 2019 & Developed acute myeloid leukemia \\
\hline Jan 2020 & Demise from acute myeloid leukemia \\
\hline
\end{tabular}

\section{Clinical Findings and Diagnostic Assessment}

Examination at our center revealed a now $25 \mathrm{~cm}$ fungating left inguinal nodal mass with adjacent $6 \mathrm{~cm}$ left upper thigh satellite nodule, with lymphedema distal to the large left groin mass (Figure 1A). This was associated with pain on movement requiring regular opioid analgesia. The patient was unable to ambulate independently due to pain and left lower limb swelling and was largely chairbound. Eastern cooperative organization group (ECOG) performance status was 2. An 18-F fluorodeoxyglucose
(FDG) positron emission tomography CT scan (PET-CT) demonstrated a fungating mass at the left groin with invasion of the adductor compartment (Figure 1C). There was also bilateral pelvic and retroperitoneal lymphadenopathy with suspicion of left lower limb lymphatic obstruction, and soft tissue deposits seen in the left gluteal region and anterior abdominal wall were consistent with metastases. Wedge biopsy of this mass at our center confirmed adenocarcinoma, clear cell subtype (Figure 1E). IHC performed demonstrated tumor expression of HNF1$\beta$, cytokeratin, CAM 5.2, but negativity for $\mathrm{p} 16$, $\mathrm{p} 40$, 


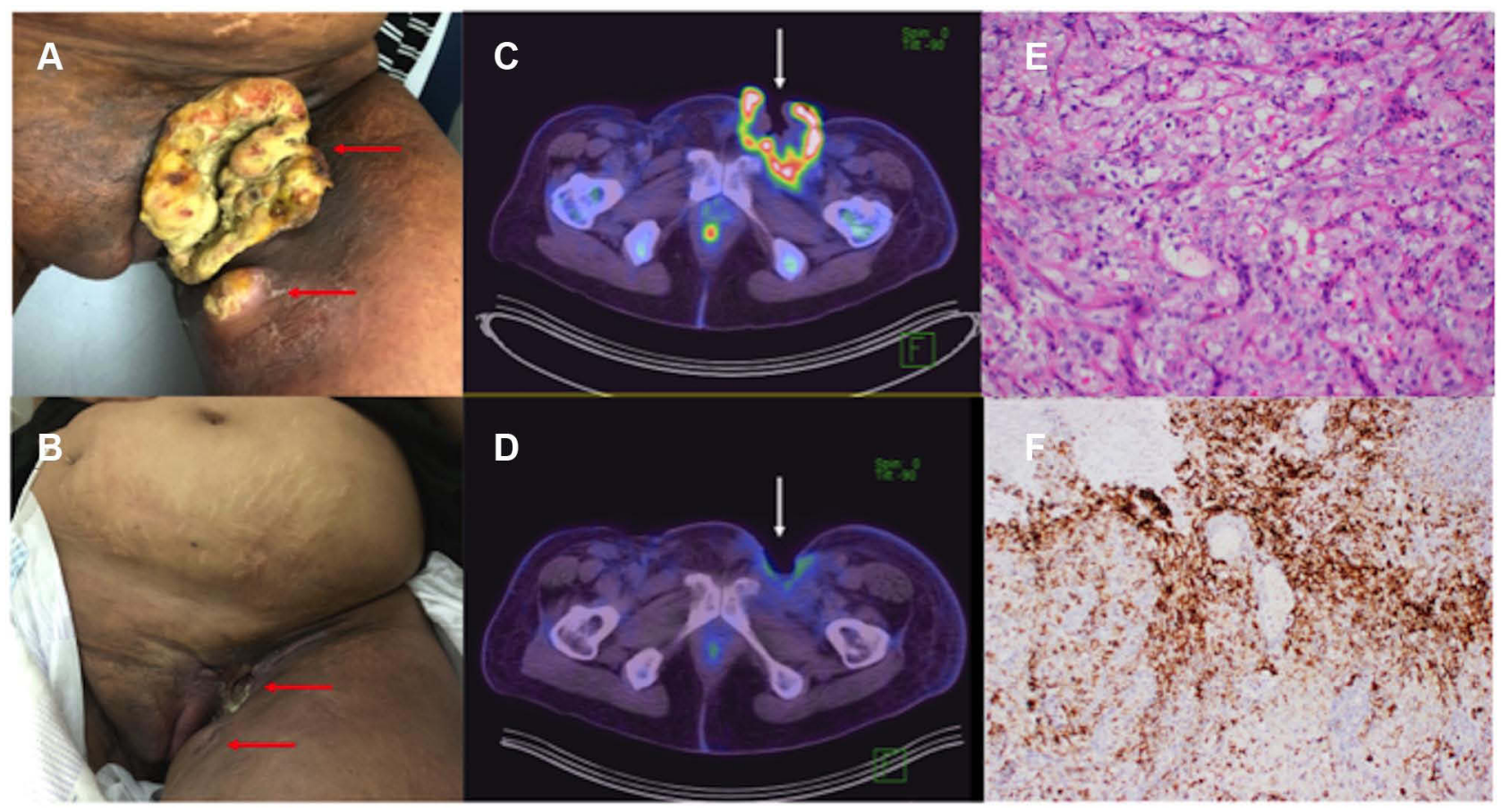

Figure I Clinical and Radiologic Response to pembrolizumab; Immunohistochemistry. (A and B) Clinical response to pembrolizumab; (A) Fungating left groin tumor and satellite nodule at baseline (September 2018), prior to starting pembrolizumab (horizontal red arrows); (B) Dramatic clinical response after 3 cycles of pembrolizumab in primary tumor and adjacent satellite nodule (December 2018) (horizontal red arrows). (C and D) Radiologic response to pembrolizumab; (C) Baseline PET-CT showing fungating and intensely FDG-avid mass (SUVmax 19.6) at the left groin (vertical white arrow) with invasion of the adductor compartment of the left thigh; (D) Follow-up PETCT after 6 months showing marked reduction in size and metabolic uptake (SUVmax $=3.9$ ) of the tumor (vertical white arrow). (E) Hematoxylin and eosin $\times 20$ stain confirming clear cell carcinoma: the tumor is composed of nests of cells with round nuclei, prominent nucleoli and ample clear to eosinophilic cytoplasm; (F) Immunohistochemistry for PD-LI using the Dako 22C3 pharmDx assay, combined positive score of 45.

napsin-A, desmin, SMA, S-100 and Melan-A. Diffuse overexpression of $\mathrm{p} 53$ on IHC was consistent with a p53mutant pattern. Expression of MLH1, MSH2, MSH6 and PMS2 was preserved. PD-L1 (Dako 22C3pharmDx) CPS was 45 (Figure 1F). The patient was enrolled on a prospective study of somatic tumor sequencing (Integrated Molecular Analysis of Cancer in Gynecologic Oncology, IMAC-GO, NCT02078544) after obtaining written informed consent. Nextgeneration tumor sequencing (FoundationOneCDx ${ }^{\circledR}$ ) showed a tumor mutational burden of 5 mutations/mega base and microsatellite-stable (MSS) status. Pathological variants were noted in $C D K N 2 A / B$ loss, TERT promoter124C $>\mathrm{T}, \quad$ TP53 G154S, MET amplification (Table 2).

\section{Therapeutic Intervention, Follow-Up and Outcomes}

In view of a high PD-L1 CPS of 45, the patient received off-label treatment with pembrolizumab $200 \mathrm{mg}$ 3-weekly from October 2018. Disease response assessment was performed by FDG PET-CT every 3 cycles, using Response Evaluation Criteria in Solid Tumors (RECIST) version 1.1. Adverse events were graded using Common Terminology Criteria for Adverse Events (CTCAE) version 5.0. The patient was adherent to treatment from October 2018 to September 2019.

Complete clinical response (Figure 1A and $\mathrm{B}$ ) and RECISTv1.1 PR on PET-CT scan (Figure 1C and D) was seen after 3 cycles of pembrolizumab. This partial response was sustained for 8 months duration. CA125 reduced from 298 to a nadir of 5.2 (Figure 2). Importantly, there was rapid resolution of her left limb pain, allowing her to discontinue all analgesia, as well as marked improvement to the associated lymphedema. Her ECOG performance status improved to 1 and she resumed independent ambulation. From our patient's perspective, she experienced a remarkable reduction in morbidity and regained her independence. Repeat biopsy of a residual sinus in the left groin showed only suppurative necrotic material. The patient experienced grade 1 fatigue and grade 1 diarrhea related to pembrolizumab that were managed supportively. However, after the fourth cycle of 
Table 2 Molecular Profiling of Pre-Pembrolizumab Tumor Biopsy

\begin{tabular}{|c|c|}
\hline Gene & Variant \\
\hline \multicolumn{2}{|c|}{ Pathogenic variants } \\
\hline MET & Amplification - equivocal \\
\hline CDKN2A/B & Loss \\
\hline TERT & Promoter - $124 \mathrm{C}>\mathrm{T}$ \\
\hline TP53 & GI54S \\
\hline \multicolumn{2}{|c|}{ Variants of uncertain significance } \\
\hline$A B L I$ & T984M \\
\hline KDR & AlI $68 \mathrm{~S}$ \\
\hline ALK & VI63L \\
\hline MLL3 & M69L \\
\hline BRCA2 & $\mathrm{R} 2896 \mathrm{H}$ \\
\hline RBI & P29del \\
\hline FGFR2 & Rearrangement \\
\hline RUNXITI & RIOT \\
\hline GPRI24 & $\mathrm{CII96Y}$ \\
\hline SPEN & V21191 \\
\hline IGFIR & P842S \\
\hline TSC2 & PI450R \\
\hline
\end{tabular}

pembrolizumab, she developed a new grade 3 neutropenia which was concerning for immune-related neutropenia. This prompted a Hematology consult, bone marrow biopsy and examination. Unexpectedly, bone marrow examination showed a normocellular marrow with dysplastic features, confirming a diagnosis of myelodysplastic syndrome with excess blasts subtype. Chromosomal analysis on her bone marrow biopsy showed clonal translocation. ${ }^{7,11}$ No clear cell carcinoma infiltration of the bone marrow was found. The diagnosis made was secondary myelodysplastic syndrome with excess blasts (MDS-EB), which was thought to be related to her previous exposure to multiple lines of cytotoxic chemotherapy. As her VCCC tumor was responding to treatment at this point, she was continued on pembrolizumab, but granulocyte colony stimulating factor (G-CSF) was avoided in view of the diagnosis of MDS-EB.

After receiving 8 months of pembrolizumab, radiological disease progression was detected on serial PET-CT. As the patient remained clinically well and asymptomatic, pembrolizumab was continued in view of continued clinical benefit. Unfortunately, after a duration of 11 months of pembrolizumab therapy, her neutropenia continued to worsen (Figure 2). This coincided with VCCC disease progression, presenting with recurrence of left groin pain and radiological findings of increased metabolic activity in the left inguinal ulcerative lesion with more prominent surrounding subcutaneous tissue and increased left thigh girth likely due to lymphatic obstruction. Alongside this, her CA125 level gradually rose (Figure 2). In September 2019, the patient returned to her home country to continue treatment, and defaulted follow-up at our center. Her disease continued to progress in her home country, coinciding with the transformation of MDS to acute myeloid leukemia (AML) in December 2019. She subsequently demised of AML in January 2020. Her overall survival (OS) from time of initiation of pembrolizumab till death was 16 months.

\section{Discussion and Conclusions}

No standard therapy exists for patients with metastatic or recurrent vulvar cancers. Owing to the rarity of this disease and the lack of their inclusion on randomized clinical trials, the prognosis for patients with metastatic or recurrent vulvar cancer is invariably poor. ${ }^{10}$ A Phase II study of weekly paclitaxel in recurrent vulvar cancer reported a median progression-free survival (PFS) of only 2.6 months,${ }^{10}$ while treatment with combination chemotherapy regimens such as cisplatin and vinorelbine or bleomycin, methotrexate and lomustine, have been associated with median OS of 19 months and 7.8 months, respectively, ${ }^{11,12}$ but at the expense of significant toxicity. In a small cohort of 20 patients with advanced vulvar cancer referred to a tertiary cancer center for early-phase clinical trials, the median OS was only 4.6 months, with no partial responses observed amongst these patients. ${ }^{9}$ Amongst diagnosed vulvar cancers, the clear cell histotype is rarely reported in the literature. A single case report has previously reported on a patient with recurrent clear cell vulvar cancer who received paclitaxel and carboplatin with stable disease as best response followed by disease progression and OS of 17 months. ${ }^{1}$ Novel approaches are urgently required to improve outcomes for patients with advanced and recurrent vulvar cancer.

The lack of randomized data guiding the treatment of advanced and recurrent vulvar cancer has led to extrapolations of treatment practice from cervical cancer paradigms, given that cervical, vulvar and vaginal cancers are often 


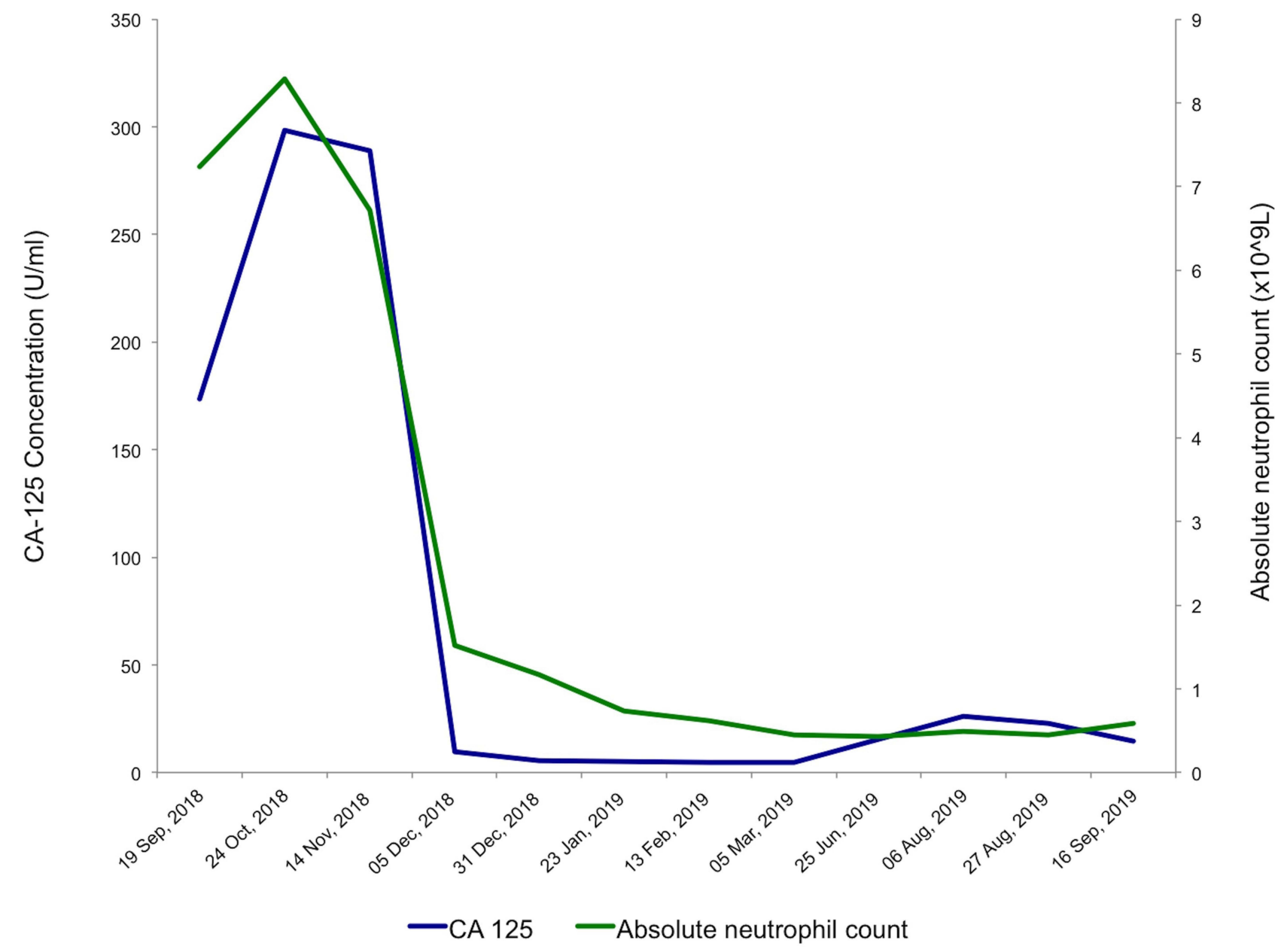

Figure 2 Relevant Biochemistry and Hematology Indices during pembrolizumab treatment. Rapid reduction in serum CA- 125 was observed after commencing pembrolizumab in October 2018, with CA-125 dropping precipitously from a baseline of $298 \mathrm{U} / \mathrm{mL}$ to $9.5 \mathrm{U} / \mathrm{mL}$ in December 2018 , after only 3 cycles of pembrolizumab. CA- 125 reached a nadir of $4.6 \mathrm{U} / \mathrm{mL}$ in February 2019. Gradual increase in CA-125 coincided with clinical disease progression in June 2019 . Worsening neutropenia occurred during the course of pembrolizumab, due to underlying myelodysplastic syndrome, which was later confirmed on bone marrow examination.

virally driven. ${ }^{7}$ Pembrolizumab has received U.S Food and Drug Administration (U.S FDA) approval for the treatment of patients with advanced or recurrent cervical cancer with disease progression after chemotherapy whose tumors express PD-L1 CPS $\geq 1$ using the U.S FDAapproved companion diagnostic. Yet, the efficacy of pembrolizumab or other anti-PD-1/PD-L1 check-point inhibitors in vulvar cancer remains poorly established. The phase I/II Checkmate-358 trial remains the only trial which has reported on a small cohort of five patients with recurrent/metastatic vaginal/vulvar cancers who were treated with nivolumab monotherapy. Of note, none of the enrolled patients were of clear cell histotype. Of these five patients, only $1 / 5$ partial responses were observed, leading to a 6-month PFS rate of $40 \%$. No correlations for response could be made with the PD-L1 status of these tumors in the study, owing to the small cohort size. Our patient represents the first described report of a patient with recurrent high PD-L1 VCCC with durable partial response to anti-PD-1 immune check-point blockade.

Despite being heavily pre-treated, our patient had a remarkable response to fifth line pembrolizumab, with PFS of 8 months and OS of 16 months. Her disease progression on pembrolizumab appeared to coincide with worsening of underlying MDS-EB. MDS is a disease of the mono-myelocytic lineage and is not expected to have a direct adverse impact on effector T-lymphocytes. It remains speculative if the development of MDS may have influenced host-tumor immune interactions and our study is limited by the lack of mechanistic investigations to study the host-tumor immune dynamics to confirm any hypothesis. Yet, the development of MDS in solid-tumor cancer patients is strongly associated with prior alkylating chemotherapy and pelvic radiation ${ }^{13}$ and it is likely that 
the development of MDS-EB was related to the multiple lines of anti-cancer therapies that she had been exposed to earlier in her disease trajectory. Earlier use of pembrolizumab in her treatment may have precluded the need for the multiple cytotoxic chemotherapies that she had received, potentially protecting her from MDS development. Therefore, this case report represents an important antecedent for the treatment of similar cases in the future.

Finally, the molecular landscape of vaginal and vulvar cancers remains poorly defined. No reports have described molecular profiling of VCCC. In our patient, the somatic mutational profile appeared to be distinct from previous reports of predominantly SCC subtype vulvar cancer whereby mutations involving the mitogen-activated protein kinase (MAPK) pathway such as KRAS (13\%) and $B R A F(14 \%)$, as well as $C$-KIT mutations (20\%) have been previously reported. ${ }^{9}$ In another case series of 15 vulvar squamous cell carcinoma (SCC) tumors, $40 \%$ of tumors harbored PIK3CA and FAT1 alterations. Copy neutral losses of heterozygosity were also noted in 7 of 15 SCCs. ${ }^{7}$ This could imply that the biological and therapeutic approach needed for VCCC may differ from that of SCC tumors.

In conclusion, our patient with VCCC harboring a high PD-L1 CPS experienced durable and meaningful response to fifth-line pembrolizumab, yet eventually demised from secondary MDS/AML, which was likely related to previous exposure to multiple lines of cytotoxic chemotherapy. The case presented here adds to the small number of case reports that address this exceedingly rare histotype of an already rare cancer, and represents the first reported case of successful treatment of VCCC with pembrolizumab. Our patient's impressive response points to the potential for immunotherapeutic exploitation in this subgroup, indicating a pressing need for further studies to investigate this approach in vulvar carcinoma.

\section{Abbreviations}

AML, acute myeloid leukemia; Anti-PD1, antiprogrammed cell death-1; AUC, area under curve; cm, centimeter; CPS, combined positive score; CT, computed tomography; CTCAE, Common Terminology Criteria for Adverse Events; ECOG, Eastern Cooperative Organization Group; FDG, fluorodeoxyglucose; FIGO, International Federation of Gynecology and Obstetrics; G-CSF, granulocyte colony stimulating factor; HNF1- $\beta$, hepatocyte nuclear factor-1 beta; IHC, immunohistochemistry; IMAC-GO, Integrated Molecular Analysis of Cancer in
Gynecologic Oncology; IV, intravenous; MDS-EB, myelodysplastic syndrome with excess blasts; Melan-A, melanoma antigen; MRI, magnetic resonance imaging; MSS, microsatellite-stable; OS, overall survival; PD-L1, programmed death-ligand 1; PET-CT, positron emission tomography CT scan; PFS, progression-free survival; PR, partial response; RECIST, Response Evaluation Criteria in Solid Tumors; SCC, squamous cell carcinoma; SMA, alpha smooth muscle actin; US FDA, United States Food and Drug Administration; VCCC, vulvar clear cell carcinoma.

\section{Data Sharing Statement}

All data generated and analyzed during this study are included in this published article.

\section{Ethics Approval and Consent to Participate}

The patient provided written consent for the publication of material relating to her condition. Ethics approval was obtained from the National Health Group Domain Specific Review Board (DSRB), Reference: 2013/00705.

\section{Consent for Publication}

Written consent for the publication of material relating to her condition was obtained from the patient.

\section{Author Contributions}

Conceptualization, M.S, NYL.N, D.L, MLM.P, YL.T, YW. L, SE.L, P.T, JHY.L, J.N, A.I, EJ.D, JJH.L, DSP.T

Data curation, M.S, NYL.N, D.L, MLM.P, YL.T, YW.L, SE.L, P.T, JHY.L, J.N, A.I, EJ.D, JJH.L, DSP.T

Writing - original draft preparation, NYL.N, M.S, D.L

Writing-review and editing, M.S, NYL.N, D.L, MLM. P, YL.T, YW.L, SE.L, P.T, JHY.L, J.N, A.I, EJ.D, JJH.L, DSP.T

Visualization, M.S, NYL.N, D.L, MLM.P, YL.T, YW.L, SE.L, P.T, JHY.L, J.N, A.I, EJ.D, JJH.L, DSP.T

Supervision, NYL.N, DSP.T, D.L.

Project administration, M.S, NYL.N, D.L, MLM.P, YL. T, YW.L, SE.L, P.T, JHY.L, J.N, A.I, EJ.D, JJH.L, DSP.T

All authors made substantial contributions to conception and design, acquisition of data, or analysis and interpretation of data; took part in drafting the article or revising it critically for important intellectual content; agreed to submit to the current journal; gave final approval of the 
version to be published; and agree to be accountable for all aspects of the work.

\section{Funding}

This study was supported by the National Medical Research Council Clinician Scientist Award Grant (NMRC/CSA-INV-0016/2017) to Dr David SP Tan and the Pangestu research fund.

\section{Disclosure}

Dr David SP Tan reports grants from National Medical Research Council, Singapore, during the conduct of the study; personal fees from Merck Sharp Dohme (MSD), grants from Astra Zeneca, Bayer, Karyopharm Therapeutics, Merck Serono, and Roche, outside the submitted work. All authors declare no other competing interests.

\section{References}

1. Buppasiri P, Kleebkaow P, Tharanon C, Aue-aungkul A, Kietpeerakool C. Clear cell carcinoma arising in vulvar endometriosis. Case Rep Pathol. 2018;2018:1-5. doi:10.1155/2018/ 4263104

2. Kojima N, Yoshida H, Uehara T, et al. Primary clear cell adenocarcinoma of the vulva: a case study with mutation analysis and literature review. Int $J$ Surg Pathol. 2019;27(7):792-797. doi:10.1177/ 1066896919848823

3. Bolis GB, Maccio T. Clear cell adenocarcinoma of the vulva arising in endometriosis. A case report. Eur J Gynaecol Oncol. 2000;21 (4):416-417.

4. Herghelegiu CG, Neacşu A, Oprescu ND, et al. Difficulties of clinical and histopathological diagnosis in advanced vulvar clear cell carcinoma. Rom J Morphol Embryol. 2018;59(4):1233-1237.
5. Messing MJ, Richardson MS, Smith MT, King L, Gallup DG. Metastatic clear-cell hidradenocarcinoma of the vulva. Gynecologic Oncology. 1993;48:264-268. doi:10.1006/gyno.1993.1045

6. Lim KC, Thompson IW, Wiener JJ. CASE REPORT A case of primary clear cell adenocarcinoma of Bartholin's gland. BJOG. 2002;109:1305-1307.

7. Han MR, Shin S, Park HC, et al. Mutational signatures and chromosome alteration profiles of squamous cell carcinomas of the vulva. Exp Mol Med. 2018;50(2):e442-13. doi:10.1038/emm.2017.265

8. Wendel Naumann R, Hollebecque A, Meyer T, et al. Safety and efficacy of nivolumab monotherapy in recurrent or metastatic cervical, vaginal, or vulvar carcinoma: results from the phase I/II CheckMate 358 trial. J Clin Oncol. 2019;37(31):2825-2834. doi:10.1200/JCO.19.00739

9. Fu S, Shi N, Wheler J, et al. Characteristics and outcomes for patients with advanced vaginal or vulvar cancer referred to a Phase I clinical trials program: the MD Anderson cancer center experience. Gynecol Oncol Res Pract. 2015;2(1):1-6. doi:10.1186/s40661-015-0018-x

10. Witteveen PO, van der Velden J, Vergote I, et al. Phase II study on paclitaxel in patients with recurrent, metastatic or locally advanced vulvar cancer not amenable to surgery or radiotherapy: a study of the EORTC-GCG (European Organisation for Research and Treatment of Cancer - Gynaecological Cancer Gro). Ann Oncol. 2009;20 (9):1511-1516. doi:10.1093/annonc/mdp043

11. Cormio G, Loizzi V, Gissi F, et al. Cisplatin and vinorelbine chemotherapy in recurrent vulvar carcinoma. Oncology. 2009;77 (5):281-284. doi:10.1159/000259259

12. Wagenaar HC, Colombo N, Vergote I, et al. Bleomycin, methotrexate, and CCNU in locally advanced or recurrent, inoperable, squamous-cell carcinoma of the vulva: an EORTC gynaecological cancer cooperative group study. Gynecol Oncol. 2001;81(3):348-354. doi:10.1006/gyno.2001.6180

13. Sun LM, Lin CL, Lin MC, Liang JA, Kao CH. Radiotherapy-and chemotherapy-induced myelodysplasia syndrome: a nationwide population-based nested case-control study. Med (United States). 2015.
OncoTargets and Therapy

\section{Publish your work in this journal}

OncoTargets and Therapy is an international, peer-reviewed, open access journal focusing on the pathological basis of all cancers, potential targets for therapy and treatment protocols employed to improve the management of cancer patients. The journal also focuses on the impact of management programs and new therapeutic

\section{Dovepress}

agents and protocols on patient perspectives such as quality of life, adherence and satisfaction. The manuscript management system is completely online and includes a very quick and fair peer-review system, which is all easy to use. Visit http://www.dovepress.com/ testimonials.php to read real quotes from published authors. 